\title{
Spin-Glass Behavior, Magnetic, and IR Spectroscopy Analysis of Multimetallic Compound $\mathrm{Ni}_{0.25} \mathrm{Mn}_{1.25}\left[\mathrm{Fe}(\mathrm{CN})_{6}\right] \cdot 6.1 \mathrm{H}_{2} \mathrm{O}$
}

\author{
Qing Lin, ${ }^{1,2}$ Xiaofang Liu, ${ }^{1}$ Yun He, ${ }^{1}$ Haifu Huang, ${ }^{1,3}$ and Xingcan Shen ${ }^{4}$ \\ ${ }^{1}$ College of Physics and Technology, Guangxi Normal University, Guilin 541004, China \\ ${ }^{2}$ Department of Information Technology, Hainan Medical College, Haikou 571101, China \\ ${ }^{3}$ Department of Physics, Nanjing University, Nanjing 210093, China \\ ${ }^{4}$ School of Chemistry and Chemical Engineering, Guangxi Normal University, 541004 Guilin, China
}

Correspondence should be addressed to Yun He; hy@gxnu.edu.cn

Received 5 June 2014; Accepted 2 July 2014

Academic Editor: Qingrui Zhang

Copyright (C) 2015 Qing Lin et al. This is an open access article distributed under the Creative Commons Attribution License, which permits unrestricted use, distribution, and reproduction in any medium, provided the original work is properly cited.

\begin{abstract}
Multimetallic Prussian blue compound $\mathrm{Ni}_{0.25} \mathrm{Mn}_{1.25}\left[\mathrm{Fe}(\mathrm{CN})_{6}\right] \cdot 6.1 \mathrm{H}_{2} \mathrm{O}$ has been prepared by coprecipitation. The temperaturedependent magnetic susceptibilities show the magnet transition for the compound at $8.5 \mathrm{~K}$. According to DC variable-temperature magnetic susceptibility paramagnetic Curie temperature $\theta$ is $-9.32 \mathrm{~K}$. The observed value of coercive field (Hc) and the remanent magnetization (Mr) for the compound are $0.32 \mathrm{KOe}$ and $0.36 \mu_{\mathrm{B}}$. According to study of zero-field-cooled (ZFC) and field-cooled (FC) magnetization curves and AC magnetization curves, there exists a spin-glass behaviour in the compound, which exhibits freezing temperature $T_{g}=7.76 \mathrm{~K}$, below magnetic transition $T_{C}=8.5 \mathrm{~K}$; that glass behavior is termed "reentrant" spin glass.
\end{abstract}

\section{Introduction}

Recently, molecule-based magnets, which can be synthesized by chemical process and have a main property of supramolecular structure, exhibit magnetic properties due to magnetic exchange interaction between magnetic ions [1-3]. The design and synthesis of molecule-based magnets have become one of the research foci on the physics and chemistry. Ones have attracted extensive attention recently owe to the high $T_{C}$ molecular ferromagnets had been discovered and the molecule-based magnets show a kinds of fascinating magnetic phenomenon such as photomagnetic effect, thermal induced magnetic properties, and magneticpole reversal which have important potential application [4-6]. Among various types of molecule-based magnetism materials, Prussian blue analogues $\mathrm{A}_{\mathrm{P}}\left[\mathrm{B}(\mathrm{CN})_{6}\right]_{q} \cdot x \mathrm{H}_{2} \mathrm{O}$ [4] (molecular structure of Prussian blue analogue compound as shown in Figure 1) play an important role due to their special structure and outstanding magnetic properties as moleculebased magnets. The multimetal Prussian blue compound $\mathrm{Ni}_{0.25} \mathrm{Mn}_{1.25}\left[\mathrm{Fe}(\mathrm{CN})_{6}\right] \cdot 6.1 \mathrm{H}_{2} \mathrm{O}$ was synthesized and has been studied for its magnetic properties through elemental analysis, IR, Mossbauer spectrum, magnetic measurements, and so forth.

\section{Experimental}

2.1. Materials and Physical Measurements. $\mathrm{NiCl}_{2} \cdot 6 \mathrm{H}_{2} \mathrm{O}$, $\mathrm{Mn}\left(\mathrm{SO}_{4}\right)_{2} \cdot 6 \mathrm{H}_{2} \mathrm{O}$, and $\mathrm{K}_{3} \mathrm{Fe}(\mathrm{CN})_{6}$ are of reagent grade and without further purification. Elemental analyses $(\mathrm{C}, \mathrm{H}$, and $\mathrm{N}$ ) were performed on Perkin-Elmer 2400 II analyser. IR spectrum was recorded on a Perkin-Elmer FT-IR spectrophotometer as $\mathrm{KBr}$ pellet in the $4000 \sim 400 \mathrm{~cm}^{-1}$ range. Magnetization measurements were measured by a Quantum Design MPMS-7S superconducting quantum interference device (SQUID) magnetometer in the scope of 2-300 K.

2.2. Synthesis of $\mathrm{Ni}_{0.25} \mathrm{Mn} n_{1.25}\left[\mathrm{Fe}(\mathrm{CN})_{6}\right] \cdot 6.1 \mathrm{H}_{2} \mathrm{O}$. Polycrystalline samples of $\mathrm{Ni}_{0.25} \mathrm{Mn}_{1.25}\left[\mathrm{Fe}(\mathrm{CN})_{6}\right] \cdot 6.1 \mathrm{H}_{2} \mathrm{O}$ have been 


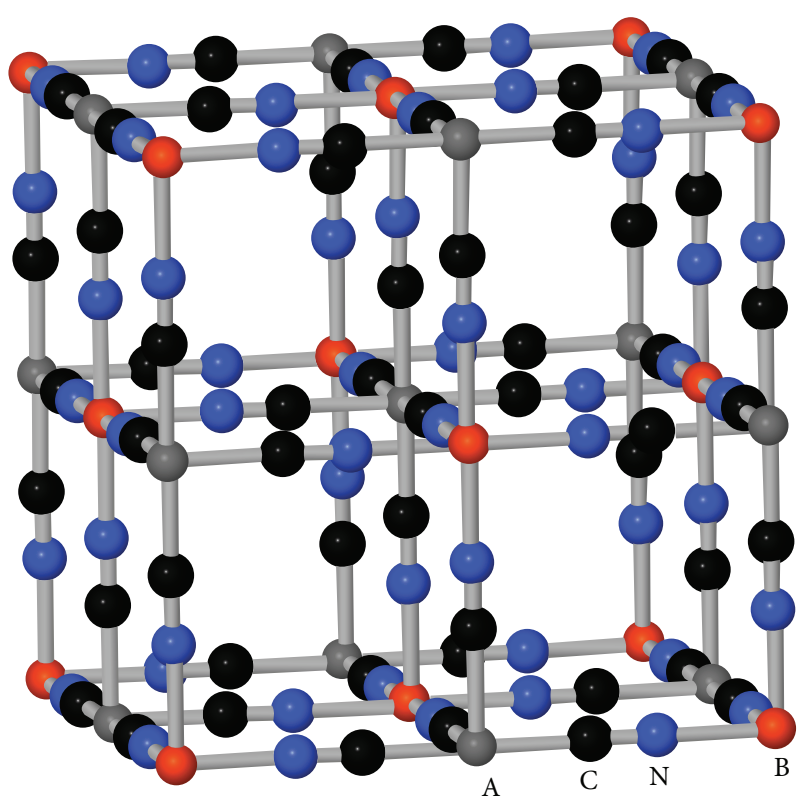

Figure 1: Structure of Prussian blue analog $\mathrm{A}_{\mathrm{P}}\left[\mathrm{B}(\mathrm{CN})_{6}\right]_{\mathrm{q}} \cdot x \mathrm{H}_{2} \mathrm{O}$.

prepared in coprecipitation method. A mixture of aqueous solutions of $\mathrm{Co}\left(\mathrm{NO}_{3}\right)_{2}(25 \mathrm{~mL}, 0.25 \mathrm{mmol})$ and $\mathrm{NiSO}_{4}$ $(125 \mathrm{~mL}, 1.25 \mathrm{mmol})$ was poured in aqueous solution of $\mathrm{K}_{3}\left[\mathrm{Fe}(\mathrm{CN})_{6}\right](100 \mathrm{~mL}, 1 \mathrm{mmol})$. Then the mixture solution was left to stand at room temperature for an appropriate period of time until those reactants were finished. A light brown precipitation was obtained, and precipitation then was filtered, washed many times with demineralized water, and finally dried under IR lamp for about 50 minutes. Elemental analysis to measure $\mathrm{C}, \mathrm{H}$, and $\mathrm{N}$ mass ratio: found: $\mathrm{C}, 17.44 \%$; $\mathrm{H}, 3.09 \%$; N, 21.23\%; calculation: C, $17.79 \%$; H, 3.03\%; N, $20.74 \%$.

\section{Results and Discussion}

3.1. IR Spectrum Analysis. IR spectrum of the compound has been recorded over the $400-4000 \mathrm{~cm}^{-1}$ range shown in Figure 2. It shows two obvious bands at 2075.01 and $2151.74 \mathrm{~cm}^{-1}$ indicating the existence of two types of cyanide groups in the crystal lattice of compound [7-9]. Compounds with $\mathrm{CN}^{-}$functional group are easily identified by their stretching frequencies in $2200-2000 \mathrm{~cm}^{-1}$ range, which are consistent with the formation of bridging cyanide groups, and there are two different coordination environments. Moreover, the broad peaks at $3432.90 \mathrm{~cm}^{-1}$ and $1615.02 \mathrm{~cm}^{-1}$ are assigned to the $v(\mathrm{O}-\mathrm{H})$ of the crystal water stretching vibrations.

3.2. DC Magnetic Susceptibility. The magnetic susceptibility of the compound was measured from $2 \mathrm{~K}$ to $300 \mathrm{~K}$ in $250 \mathrm{Oe}$ field. Figure 3 shows the field-cooled magnetization $(M)$ versus temperature $(T)$ curve and a sharp increase in $M$ is observed around $21 \mathrm{~K}$. Magnetic transition temperature was estimated from minima of $d M / d T$ versus $T$ curve, which

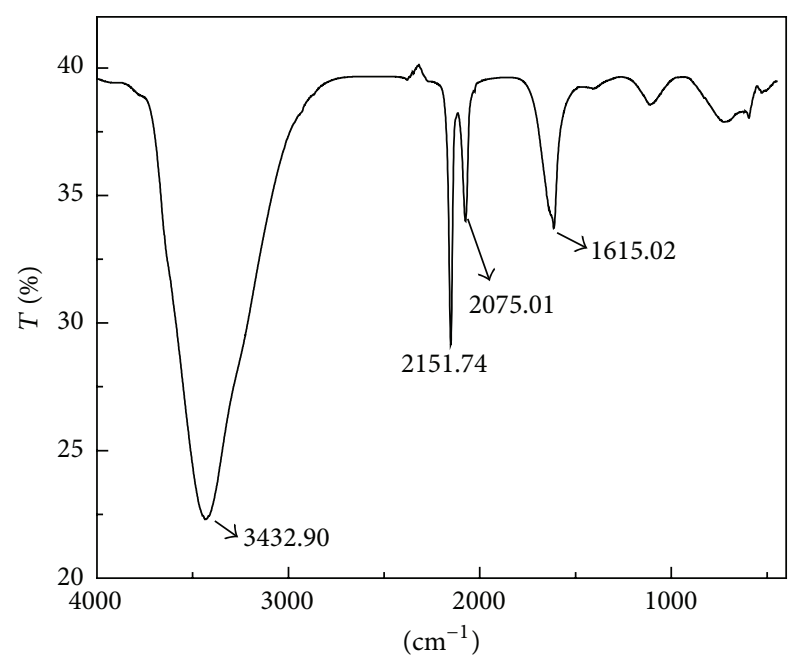

FIGURE 2: FT-IR spectrum of the compound.

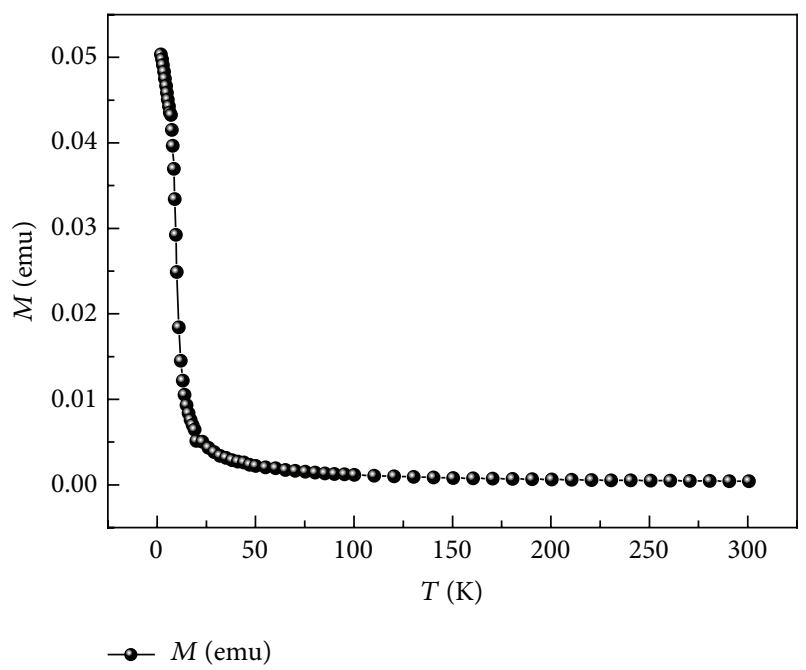

Figure 3: $M$ versus $T$ for the compound.

corresponds to the steepest increase of magnetization with decreasing temperature (as shown in Figure 4). The phase transition the compound undergoes from a paramagnetic to ferro/ferrimagnetic type is about $8.5 \mathrm{~K}$, which is lower than that for the parent compound $\mathrm{Ni}_{1.5}\left[\mathrm{Fe}(\mathrm{CN})_{6}\right] \cdot x \mathrm{H}_{2} \mathrm{O}\left(T_{C}=\right.$ $23.6 \mathrm{~K})[10]$.

The inverse susceptibility as a function of temperature in the paramagnetic state is shown in Figure 5. The curve rises slowly with decrease of temperature from 300 to $25 \mathrm{~K}$ and then rises sharply as temperature continues to decrease. The $\chi_{m}$ shows a sharp maximum at $2 \mathrm{~K}$. This kind of behaviour is a characteristic of a ferromagnet. The magnetic order results from the combination of ferromagnetic and neighboring antiferromagnetic interactions. Furthermore, high temperature DC susceptibility $\left(\chi_{m}=M / H\right)$ is found to obey the Curie-Weiss law.

Figure 6 shows the temperature dependence of $\chi_{m}{ }^{-1}$ in the temperature range of $20-280 \mathrm{~K}$. The Curie constant $(C)$ 


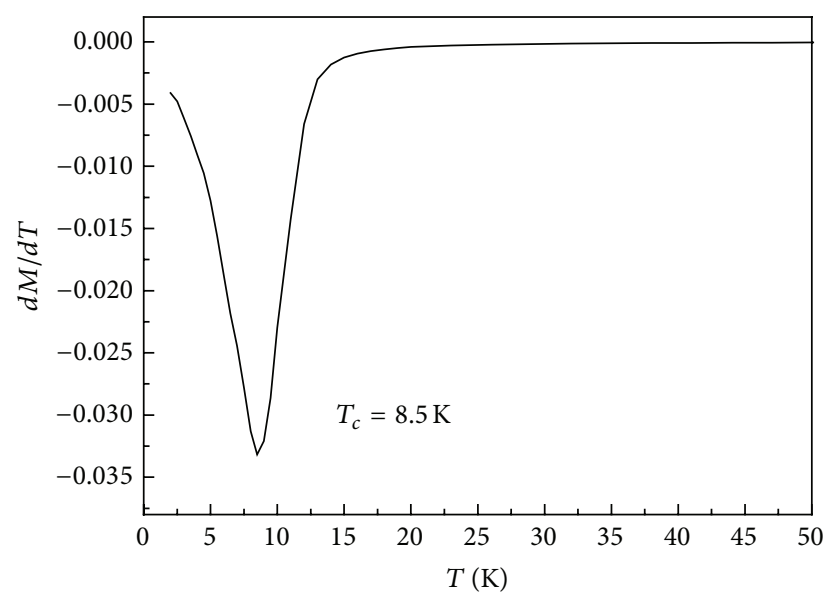

Figure 4: $d M / d T$ versus $T$ for the compound.

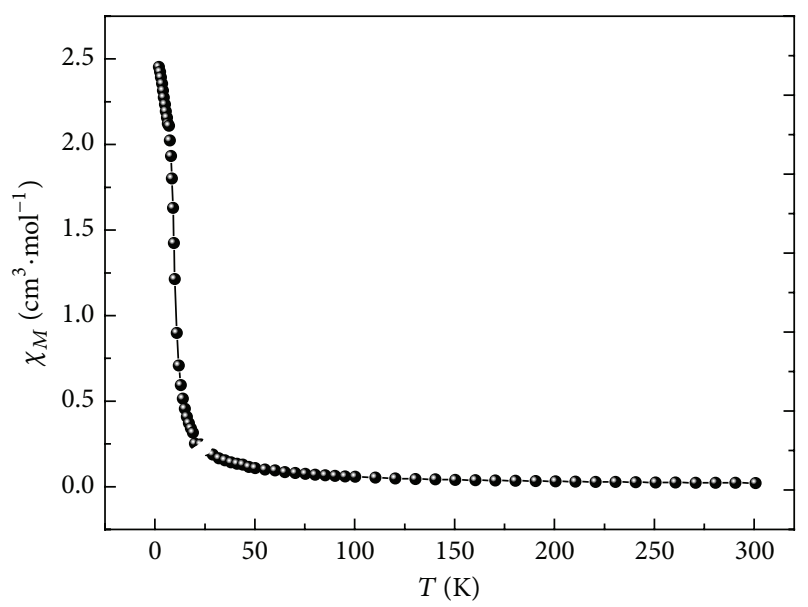

FIgURE 5: $\chi_{m}$ versus $T$ for the compound.

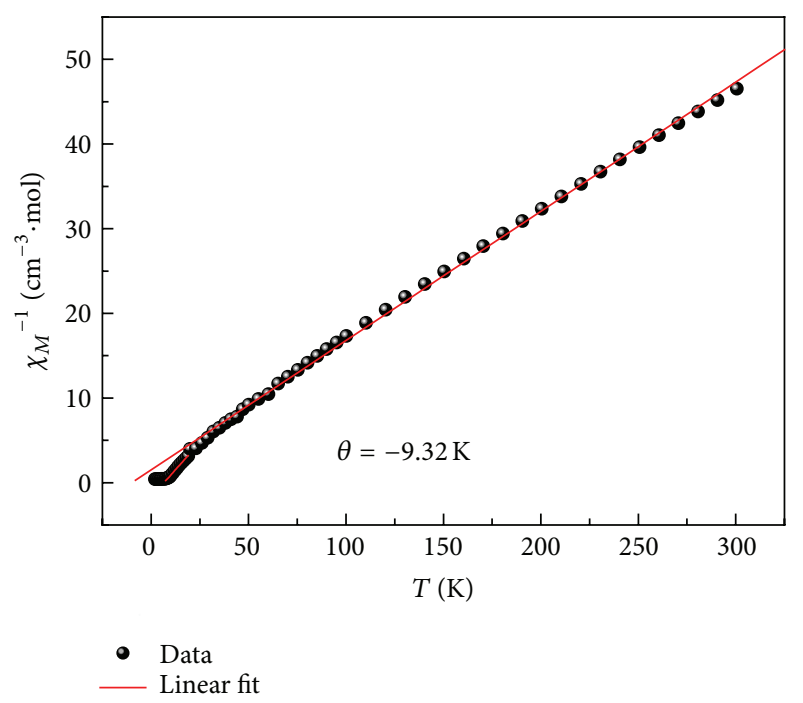

Figure 6: $\chi_{m}{ }^{-1}$ versus $T$ for the compound.

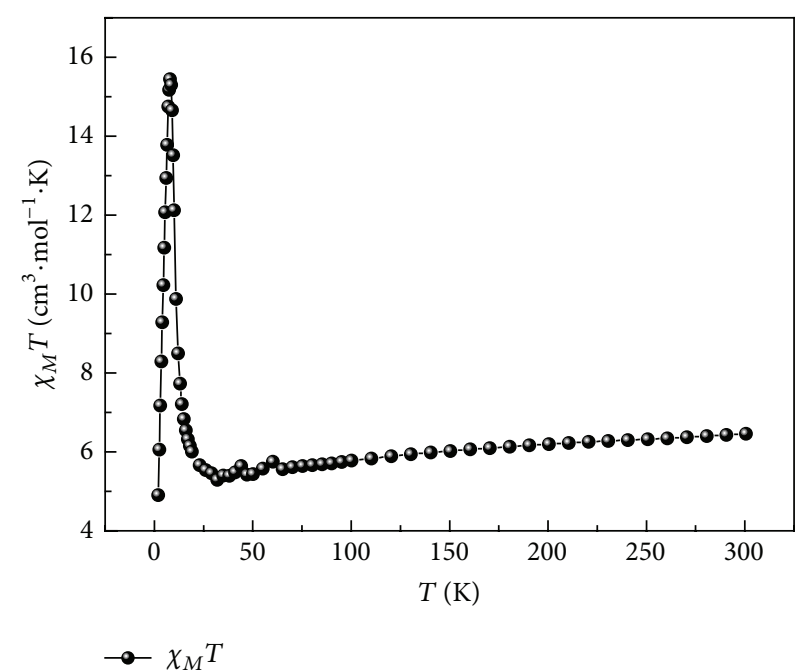

FIgURE 7: $\chi_{m} T$ versus $T$ for the compound.

and the Curie-Weiss temperature $(\theta)$ are estimated by a linear fitting of $1 / \chi=(T-\theta) / C$ at the linear region [8-10]. Fitting yielded that the Curie constant $C=15.20 \mathrm{~cm}^{3} \cdot \mathrm{K} \cdot \mathrm{mol}^{-1}$ and paramagnetic Curie temperature $\theta=-9.32 \mathrm{~K}$. The values of $T_{C}, \theta$, and $C$ are different from those values for ferrimagnet $\mathrm{Ni}_{1.5}\left[\mathrm{Fe}(\mathrm{CN})_{6}\right] \cdot x \mathrm{H}_{2} \mathrm{O}[10]$ and $\mathrm{Mn}_{3}\left[\mathrm{Fe}(\mathrm{CN})_{6}\right]_{2} \cdot 15 \mathrm{H}_{2} \mathrm{O}\left(T_{C}=\right.$ $9 \mathrm{~K})$ [11].

A curve of $\chi_{m} T$ versus $T$ is shown in Figure 7 , and the $\chi_{m} T$ value at room temperature is $5.7 \mathrm{~cm}^{3} \cdot \mathrm{K} \cdot \mathrm{mol}^{-1}$. Upon lowering the temperature, $\chi_{m} T$ value sharply increases after $15 \mathrm{~K}$ with a further decrease of the temperature. The $\chi_{m} T$ shows a sharp maximum value of $35.3 \mathrm{~cm}^{3} \cdot \mathrm{K} \cdot \mathrm{mol}^{-1}$ at $9 \mathrm{~K}$ and then finally decreases more rapidly on further cooling. For a ferromagnetic compound, $\chi_{m} T$ versus $T$ curve reaches a minimum before rising around magnetic ordering temperature [12-14].

A curve of $\mu_{\text {eff }}$ versus $T$ is shown in Figure 8. The effective moment $\mu_{\text {eff }}$ first slowly decreases to reach a minimum of $6.47 \mu_{\mathrm{B}}$ at $23 \mathrm{~K}$ and then sharply increases to reach maximum of $16.83 \mu_{\mathrm{B}}$ at $9 \mathrm{~K}$ and final decrease at lower temperature, indicating antiferromagnetic interaction between paramagnetic centers [15-17]. Magnetic transition temperature was estimated from minimum of $d \mu_{\mathrm{eff}} / d T$ versus $T$ curve, which corresponds to the steepest increase of magnetization with decreasing temperature (as shown in Figure 6; insert: plot of $d \mu_{\text {eff }} / d T$ versus $T$ ). The compound undergoes a paramagnetic to ferro/ferrimagnetic type phase transition at $9.5 \mathrm{~K}$, which could be attributed to an intermolecular antiferromagnetic interaction and/or a zero-field splitting (ZFS) effect. This kind of behaviour is a characteristic of a ferromagnet $[18,19]$.

3.3. Zero-Field-Cooled (ZFC) and Field-Cooled (FC) Magnetization. Figure 9 shows the curves of zero-field-cooled (ZFC) and field-cooled (FC) magnetization of the compound at different field $H=20,100,250$, and 500 Oe. The values of $M$ increase and exhibit weak irreversibility in 


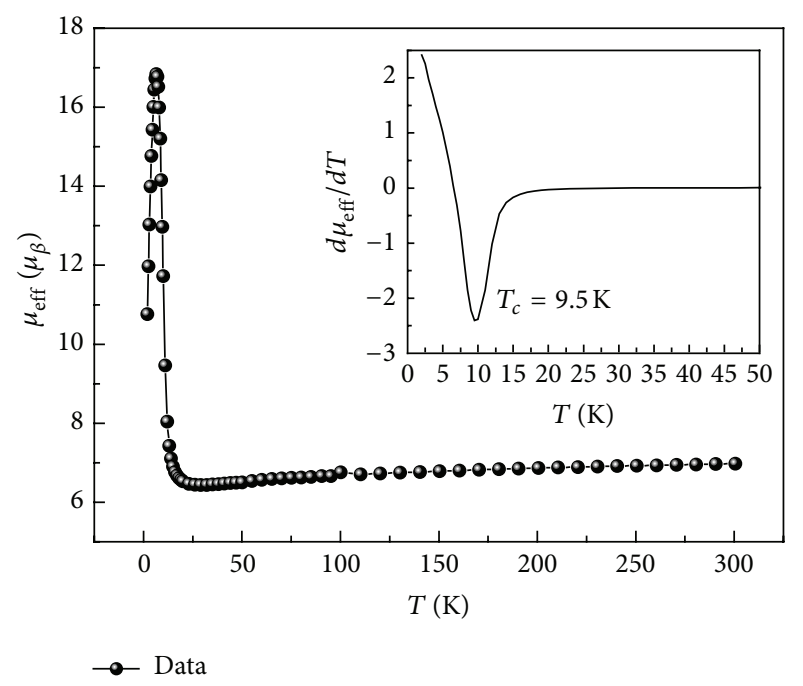

FIgURE 8: $\mu_{\mathrm{eff}}$ versus $T$ for the compound (insert: plot of $d \mu_{\mathrm{eff}} / d T$ versus $T$ ).

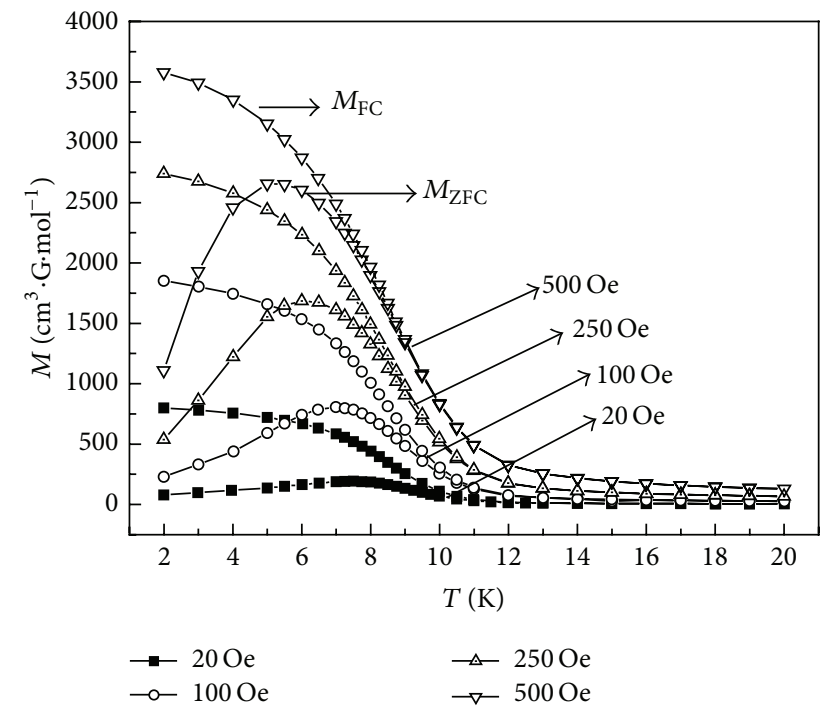

FIGURE 9: ZFC and FC magnetization curves with different field.

the field-cooled (FC) magnetization curves below $T_{C}=12 \mathrm{~K}$. There is a clear bifurcation phenomenon of the field-cooled $\left(M_{\mathrm{FC}}\right)$ and zero-field-cooled $\left(M_{\mathrm{ZFC}}\right)$ magnetization curves. $T_{\text {irr }}$ is a bifurcation temperature point of which FC and ZFC magnetization curves separate out. In addition, the behavior that $M_{\mathrm{ZFC}}$ exhibits a maximum below $T_{\text {irr }}$ is attributed to the cooperative freezing of spin glass (as shown in Figure 10). The irreversible behavior of $M$ and shift of bifurcation point to lower temperature with increasing $H$ are characteristic for spin glasses $[10,11]$. It may be reasonable that these metal ions $\mathrm{Fe}^{\mathrm{III}}, \mathrm{Ni}^{\mathrm{II}}$, and $\mathrm{Mn}^{\mathrm{II}}$ through cyanide-bridged ligand have the coexistence of different valence states or spin states, the presence of inhomogeneity, and inherent structural disorder, which propagate possibly the ferromagnetic and antiferromagnetic exchange interaction via bridging cyanide,

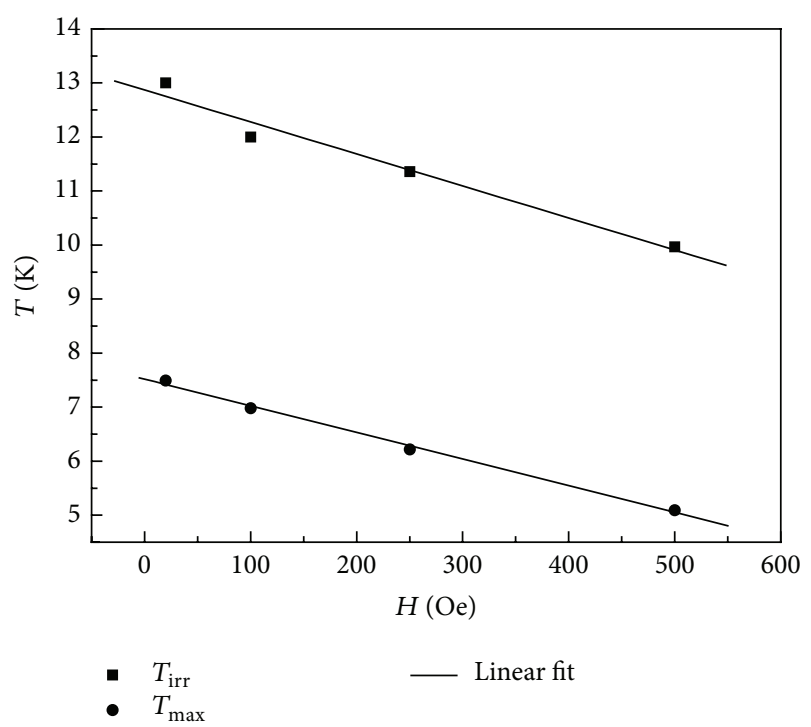

FIgURE 10: $T_{\text {irr }}$ and $T_{\max }$ versus $H$ for the compound.

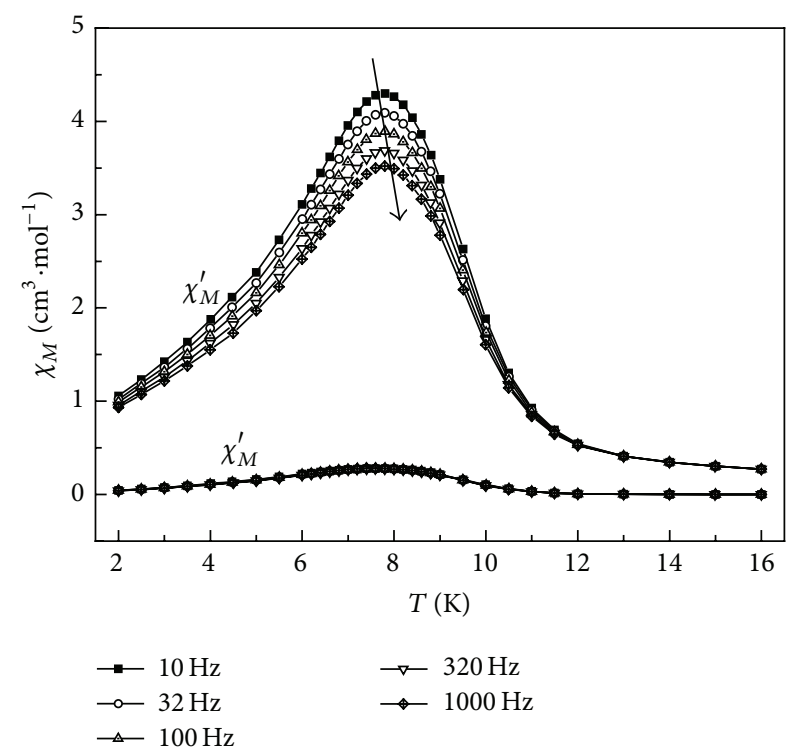

FIGURE 11: $\chi^{\prime}(T)$ and $\chi^{\prime \prime}(T)$ curves of AC magnetic susceptibility of the compound with different frequencies.

and there exist a structural disorder and a certain content of crystallization water which lead to the weak spin-orbit coupling. The spin-glass property is due to magnetic domain kinetics under different cooling conditions and the presence of available vacant sites in the lattice for the water molecules.

3.4. AC Magnetic Susceptibility. It was also confirmed that there exists a spin-glass behavior in the compound through AC magnetization curves. The AC magnetic susceptibility of the compound was measured at $4 \mathrm{Oe} \mathrm{AC}$ amplitude with zero-applied DC field when varying the frequencies $(f)$ from 10 to $1000 \mathrm{~Hz}$, as shown in Figure 11. The temperature dependence of zero-static field AC magnetic susceptibilities shows 


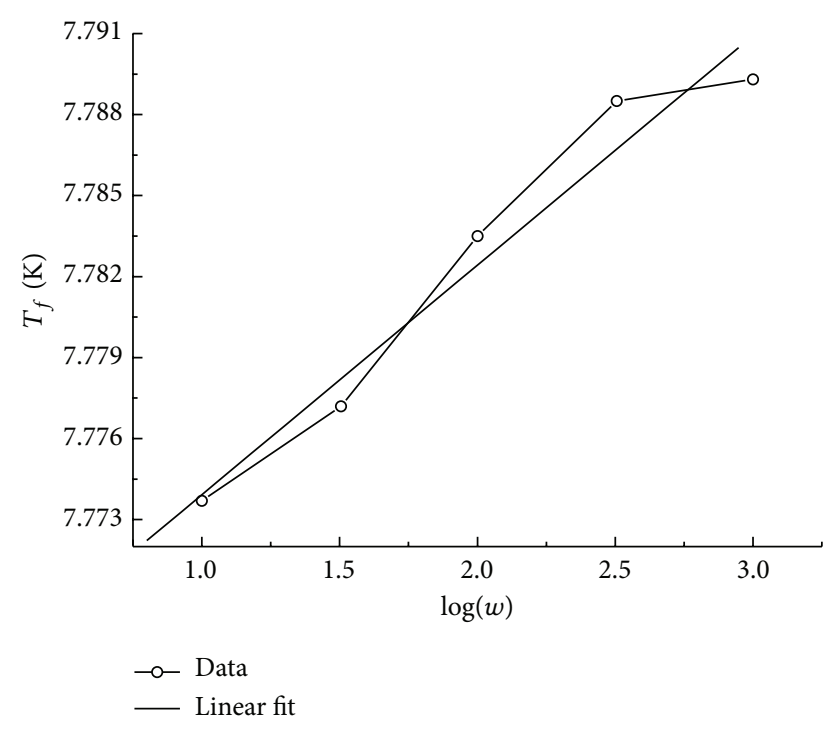

Figure 12: The $\log (w)$ dependence of the $T_{f}$.

that the in-phase component $\left(\chi^{\prime}\right)$ has a maximum at about $7.7 \mathrm{~K}$ for frequencies of $10,32,100,320$, and $997 \mathrm{~Hz}$ and that a significant out-of-phase component $\left(\chi^{\prime \prime}\right)$ appears, confirming the long-range ferromagnetic ordering. On decreasing temperature, the in-phase signals $\chi^{\prime}$ increase abruptly at around $12 \mathrm{~K}$, reach the maximum at about $7.7 \mathrm{~K}$, and then decrease slowly. The out-of-phase signals $\chi^{\prime \prime}$ increase steadily to the maximum around $8 \mathrm{~K}$ and then decrease slowly, as shown in Figure 11. The fact that the $\chi^{\prime}(T)$ clearly shows a frequency dependence is typically assigned to spin glasses [12, 13]. The freezing temperature $\left(T_{f}\right), T_{f}=7.7 \mathrm{~K}$, is defined by the maximum in the $x_{\mathrm{AC}}(T)$ plot at low frequency. It undergoes a paramagnetic to ferromagnetic transition at around $7.7 \mathrm{~K}$. In fact, the temperature value of the maximum of $\chi$ at a given frequency $(n)$ corresponds to the blocking temperature $\left(T_{N}=T_{\max }\right)$, whereby it is assumed that the switching of the oscillating AC field matches the relaxation rate of the magnetization.

Proportional relationship between freezing temperature $T_{f}$ and logarithm of frequency in spin-glass system can be described by quantifying the frequency dependence through the ratio $c$, which can be written as $c=\Delta T_{f} / T_{f} \Delta \log w$. $T_{f}(w)$ versus $\log (w)$ is shown in Figure 12. The value of freezing temperature of zero frequency is $T_{g}=7.76 \mathrm{~K}$ by extrapolation method and the value of $c$ obtained for the compound is 0.0011 , which fall within the range typical for the conventional spin-glass system $\left(10^{-2}-10^{-3}\right)$. Surprisingly, both the in-phase and out-of-phase signals $\left(\chi^{\prime}\right.$ and $\left.\chi^{\prime \prime}\right)$ go through a maximum with strong frequency dependence. Both in the real and in the imaginary components the peaks shift to lower temperatures with decreasing frequencies. However, the intensities of the peaks behave differently. While the intensity of the peaks for the real component increases with decreasing frequencies, in the imaginary component the intensity of the peaks decreases with decreasing frequencies.

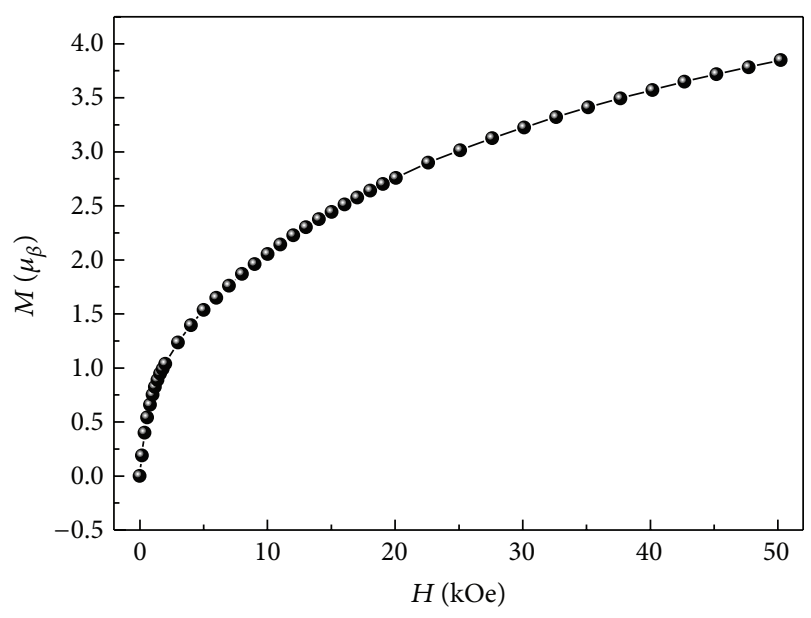

FIGURE 13: Field-dependent magnetization curves.

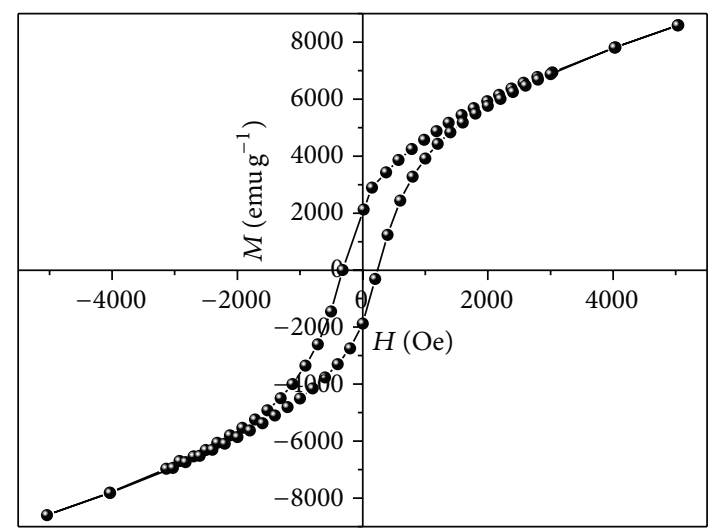

FIgURE 14: The hysteresis loop for the compound.

This behaviour of $\chi^{\prime \prime}$ and $\chi^{\prime \prime}$ is typical of a spin-glass state [16-19].

3.5. Field-Dependent Magnetization and Hysteresis Behavior. In order to further understand the nature of magnetic ordering, the ferromagnetism behavior is characterized by the measurements of field-dependent magnetization, as shown in Figures 11 and 13. The observed $M_{s}$ value is $3.85 \mu_{\mathrm{B}}$ at $50 \mathrm{kOe}$, but the compound does not reach full saturation and this behavior is likely to be related to the spinglass behavior as well as the amount of spin and type of coupling in the compounds $\mathrm{Ni}_{1.5}\left[\mathrm{Fe}(\mathrm{CN})_{6}\right] \cdot x \mathrm{H}_{2} \mathrm{O}$ [18] and $\mathrm{Mn}_{3}\left[\mathrm{Fe}(\mathrm{CN})_{6}\right]_{2} \cdot 15 \mathrm{H}_{2} \mathrm{O}[10]$.

Ferromagnetism in the compound $\mathrm{Ni}_{0.25} \mathrm{Mn}_{1.25}\left[\mathrm{Fe}(\mathrm{CN})_{6}\right]$. $6.1 \mathrm{H}_{2} \mathrm{O}$ is also supported by hysteresis loop curves measured at $4 \mathrm{~K}$ as shown in Figure 14. The coercive field $\left(H_{c}\right)$ was $0.32 \mathrm{kOe}$, which was smaller than that of the compound $\mathrm{Ni}_{1.5}\left[\mathrm{Fe}(\mathrm{CN})_{6}\right] \cdot x \mathrm{H}_{2} \mathrm{O}\left(H_{c}=2.5 \mathrm{KOe}, 4.4 \mathrm{~K}\right)$ [12]. The remanent magnetization $\left(M_{r}\right)$ with $0.32 \mu_{\mathrm{B}}$ for the compound. It is also obtained by the hysteresis loop curves.

Therefore, synthesis ideas of molecular alloy magnet can be regarded as a synthesis method to expand a new type of magnetic functional materials, whose magnetic properties 
can be tuned and controlled by changing the composition of different transition metal cations [15, 17-19].

\section{Conclusion}

We have reported a detailed investigation of magnetic properties of multimetallic Prussian blue compounds $\mathrm{Ni}_{0.25} \mathrm{Mn}_{1.25}\left[\mathrm{Fe}(\mathrm{CN})_{6}\right] \cdot 6.1 \mathrm{H}_{2} \mathrm{O}$. The temperature-dependent magnetic susceptibilities show the magnetism transition for the compound at $8.5 \mathrm{~K}$. The Curie constant $\left(C=15.20 \mathrm{~cm}^{3} \cdot \mathrm{K} \cdot \mathrm{mol}^{-1}\right)$ and the Curie-Weiss temperature $(\theta=-9.32 \mathrm{~K})$ are obtained through a linear fitting of $1 / \chi=(T-\theta) / C$ at the linear region [8-10].

The observed values of coercive field $\left(H_{c}\right)$ and remanent magnetization $\left(M_{r}\right)$ for the compound are $0.32 \mathrm{KOe}$ and $0.36 \mu_{B}$. Moreover, there exists a spin-glass behaviour in the compound according to study of zero-field-cooled (ZFC) and field-cooled (FC) magnetization curves and AC magnetization curves, which exhibits freezing temperature $T_{g}=7.76 \mathrm{~K}$, below magnetic transition $T_{c}=8.5 \mathrm{~K}$. Such a glass behavior is termed "reentrant" spin glass. It was also confirmed by the behaviour of $\chi^{\prime \prime}$ and $\chi^{\prime \prime}$, which go through a maximum with strong frequency dependence.

\section{Conflict of Interests}

The authors declare that there is no conflict of interests regarding the publication of this paper.

\section{Acknowledgments}

This work was financially supported by the National Natural Science Foundation of China (nos. 11164002 and 11364004) and Innovation Project of Guangxi Graduate Education under Grant no. 0991092.

\section{References}

[1] J. M. Manriquez, G. T. Yee, R. S. Mclean, A. J. Epstein, and J. S. Miller, "A room-temperature molecular/organic-based magnet," Science, vol. 252, no. 5011, pp. 1415-1417, 1991.

[2] S. Ferlay, T. Mallah, R. Ouahès, P. Veillet, and M. Verdaguer, "A room-temperature organometallic magnet based on Prussian blue," Nature, vol. 378, no. 6558, pp. 701-703, 1995.

[3] O. Sato, T. Iyoda, A. Fujishima, and K. Hashimoto, "Electrochemical tunable magnetic phase transition in a high-Tc chromium cyanide thin film," Science, vol. 271, no. 5245, pp. 4951, 1996.

[4] M. Giorgetti, "A review on the structural studies of batteries and host materials by X-ray absorption spectroscopy," ISRN Materials Science, vol. 2013, Article ID 938625, 22 pages, 2013.

[5] S.-I. Ohkoshi, T. Iyoda, A. Fujishima, and K. Hashimoto, "Magnetic properties of mixed ferro-ferrimagnets composed of Prussian blue analogs," Physical Review B Condensed Matter and Materials Physics, vol. 56, no. 18, pp. 11642-11652, 1997.

[6] S. Ohkoshi, O. Sato, T. Iyoda, A. Fujishima, and K. Hashimoto, "Tuning of superexchange couplings in a molecule-based ferroferrimagnet: $\left(\mathrm{Ni}_{x}^{I I} \mathrm{Mn}_{1-x}^{I I}\right) 1.5\left[\mathrm{Cr}^{I I I}(\mathrm{CN})_{6}\right]$," Inorganic Chemistry, vol. 36, pp. 268-269, 1997.
[7] S.-I. Ohkoshi, Y. Abe, A. Fujishima, and K. Hashimoto, "Design and preparation of a novel magnet exhibiting two compensation temperatures based on molecular field theory," Physical Review Letters, vol. 82, no. 6, pp. 1285-1288, 1999.

[8] A. Kumar and S. M. Yusuf, "Magnetic properties of multi-metal Prussian Blue analogue $\mathrm{Co}_{0.75} \mathrm{Ni}_{0.75}\left[\mathrm{Fe}(\mathrm{CN})_{6}\right] \cdot 6.8 \mathrm{H}_{2} \mathrm{O}$," Physica B: Condensed Matter, vol. 362, no. 1-4, pp. 278-285, 2005.

[9] A. Kumar, S. M. Yusuf, and L. Keller, "Neutron diffraction study of molecular magnetic compound $\mathrm{Ni}_{1.125} \mathrm{Co}_{0.375}\left[\mathrm{Fe}(\mathrm{CN})_{6}\right]$. 6.4 $\mathrm{H}_{2} \mathrm{O}$," Physica B, vol. 385-386, pp. 444-446, 2006.

[10] O. Kahn, S. J. Miller, and F. Palacio, Eds., Magnetic Molecular Materials, vol. 198 of NATO ASI Series E, Plenum, New York, NY, USA, 1991.

[11] S. Juskzyk, C. Johanssont, M. Hansont, A. Ratusmas, and G. Makckill, "Ferromagnetism of the $\mathrm{Me}_{3}\left[\mathrm{Fe}(\mathrm{CN})_{6}\right] \cdot \mathrm{H}_{2} \mathrm{O}$ compounds, where $\mathrm{Me}=\mathrm{Ni}$ and Co," Journal of Physics: Condensed Matter, vol. 6, pp. 5697-5765, 1994.

[12] J. S. Miller and A. J. Epstein, "Organic and organometallic molecular magnetic materials-designer magnets," Angewandte Chemie International Edition in English, vol. 33, no. 4, pp. 385415, 1994.

[13] O. Kahn, Molecular Magnetism, Wiley-VCH, New York, NY, USA, 1993.

[14] W. E. Buschmann and J. S. Miller, "Magnetic ordering and spinglass behavior in first-row transition metal hexacyanomanganate(IV) Prussian Blue analogues," Inorganic Chemistry, vol. 39, no. 11, pp. 2411-2421, 2000.

[15] Y. He, Y.-D. Dai, H.-B. Huang, J. Lin, and Y.-F. Hsia, "Spinglass state and ferromagnetic order in $\mathrm{Cu}(\mathrm{II})-\mathrm{Fe}(\mathrm{III})$ cyanides," Chinese Physics, vol. 13, no. 5, pp. 746-749, 2004.

[16] H. Huang, L. Wei, Y. He, X. Li, and F. Liang, "Ferromagnetic order and spin-glass behavior in multi-metallic compound $\mathrm{Ni}_{1.125} \mathrm{Co}_{0.375}\left[\mathrm{Fe}(\mathrm{CN})_{6}\right] \cdot 6.8 \mathrm{H}_{2} \mathrm{O}$," Current Applied Physics, vol. 9, no. 5, pp. 1160-1164, 2009.

[17] S.-I. Ohkoshi, S. Yorozu, O. Sato, T. Iyoda, A. Fujishima, and K. Hashimoto, "Photoinduced magnetic pole inversion in a ferro-ferrimagnet: $\left(\mathrm{Fe}_{0.40}^{\mathrm{II}} \mathrm{Mn}_{0.60}^{\mathrm{II}}\right)_{15} \mathrm{Cr}^{\mathrm{III}}(\mathrm{CN})_{6}$," Applied Physics Letters, vol. 70, no. 8, pp. 1040-1042, 1997.

[18] D. Sherrington and S. Kirkpatrick, "Solvable model of a spinglass," Physical Review Letters, vol. 35, no. 26, pp. 1792-1796, 1975.

[19] M. Gabay and G. Toulouse, "Coexistence of spin-glass and ferromagnetic orderings," Physical Review Letters, vol. 47, no. 3, pp. 201-204, 1981. 

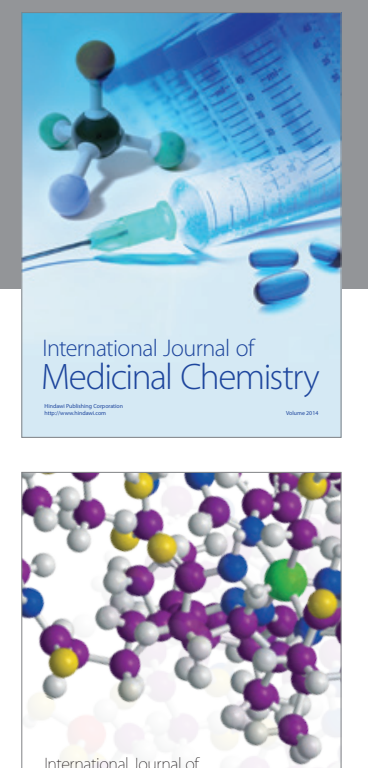

\section{Carbohydrate} Chemistry

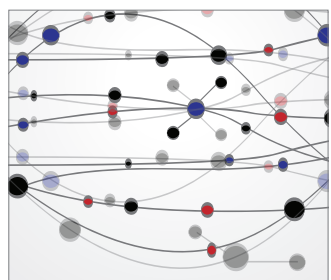

The Scientific World Journal
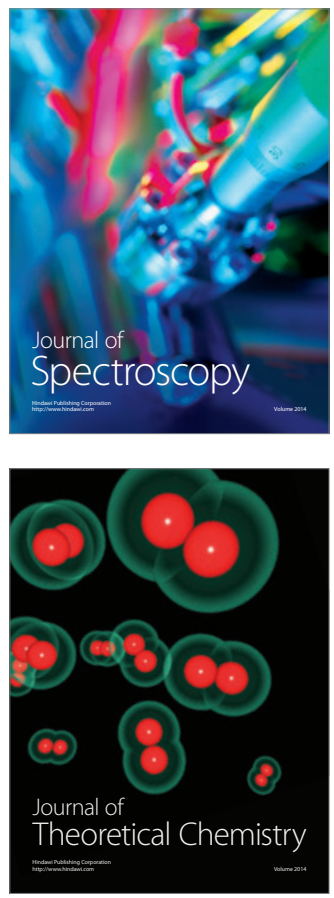
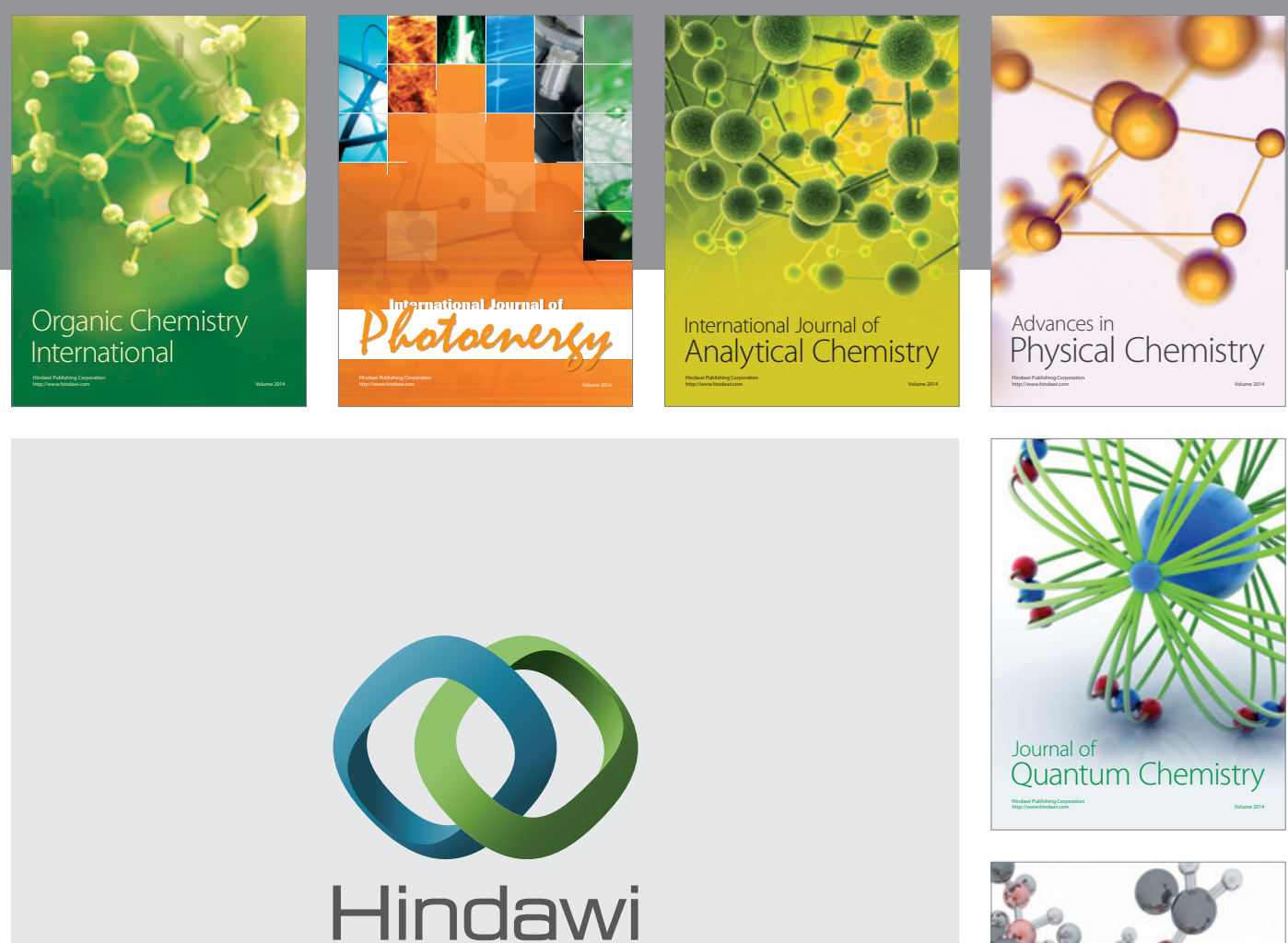

Submit your manuscripts at

http://www.hindawi.com

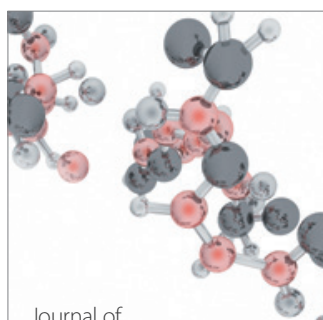

Analytical Methods

in Chemistry

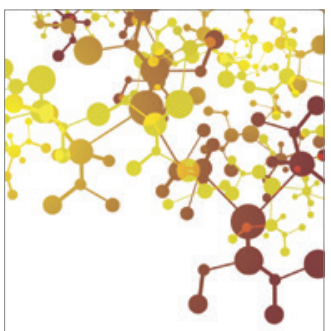

Journal of

Applied Chemistry

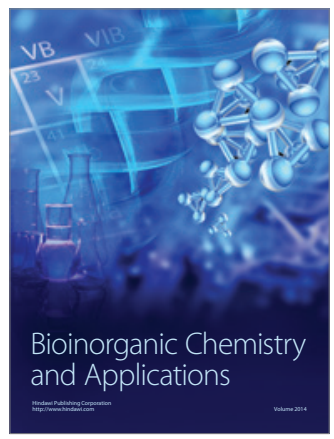

Inorganic Chemistry
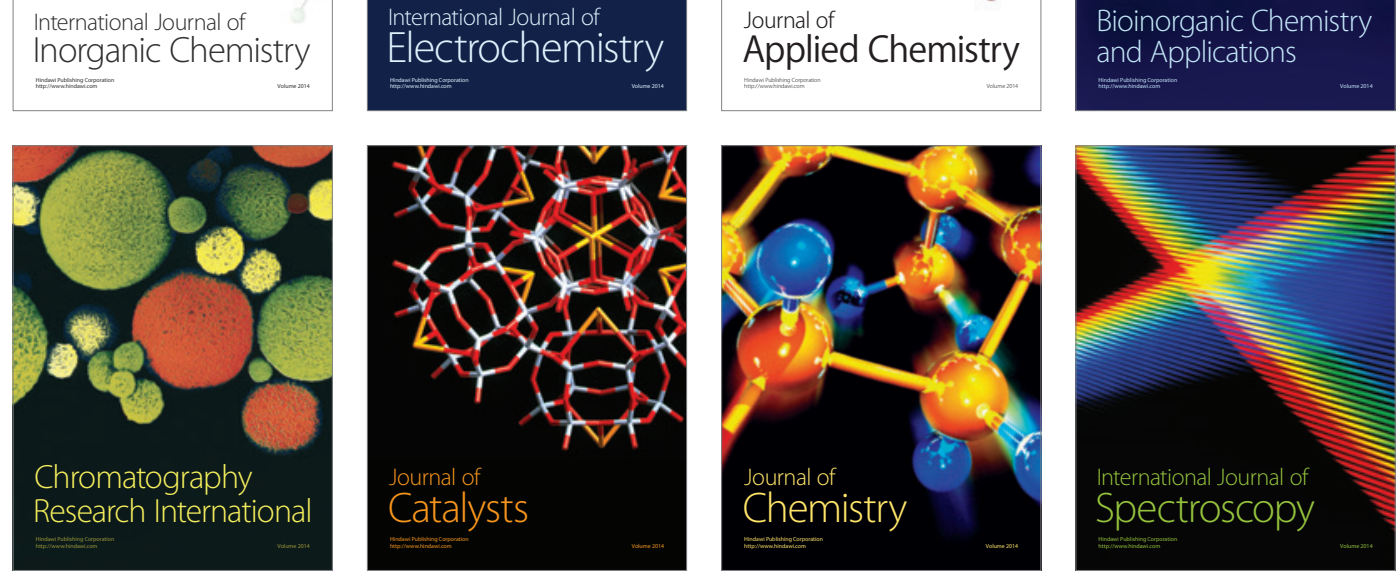